\title{
Frictional Drag between Non-Equilibrium Electron Gases
}

\author{
X. F. Wang and I. C. da Cunha Lima \\ Instituto de Física, UERJ, 22.500-013 Rio de Janeiro, RJ, Brazil
}

Received on 23 April, 2001

\begin{abstract}
Using the balance of forces in the linear regime, it is shown that the ratio between the induced voltage in an open circuit Coulomb drag experiment, and the induced current in closed circuit, equals the internal resistence of the circuit in which the induction occurs.
\end{abstract}

During the last decade much attention was given to a mechanism of current/voltage induction between two isolated systems (in the sense that momentum transfer via particles exchange is prohibited) made possible through Coulomb interaction between carriers at each of them. Such mechanism has been called Coulomb Drag (CD). The predictions made by Progrebinskii [1] and Price[2], have been confirmed later by Solomon et al [3] and, more recently, by a series of experiments involving quasi-two-dimensional systems in semiconductor double quantum wells $[4,5]$. Placing two 2Ddimensional electron systems close to each other, and separated by a potential barrier, when an electromotive force is applied in one of them, an induced voltage is observed in the other in an open circuit condition, or an induced current will appear, in the case of closed circuit. In linear regime, a transresistivity is defined as the ratio between the induced electric field and the driving current density. Improvements, like the inclusion of phonons drag, indirect Coulomb interaction by virtual phonons, usage of field theoretical techniques, etc., contributed to the fast development of this subject in recent years.

In general, experiments and calculations are performed in a open circuit set-up, since the existence of a current in the induced circuit makes the calculation much more complicate. In this work we used the Balance Equations formalism [6] to study, in the linear regime of a $\mathrm{CD}$ experiment, the relation between induction of voltage and induction of current in open and closed circuit situations. Lets $\mathbf{v}_{1}$ and $\mathbf{v}_{2}$ denote the drift velocities of the two systems, $T_{1}$ and $T_{2}$ their quasi-equilibrium temperatures, $n_{1}$ and $n_{2}$ their electron densities, and $\mathbf{E}_{1}$ and $\mathbf{E}_{2}$ the respective drifting and induced electric fields. The balance of force equations [7]:

$$
\begin{aligned}
& -n_{1} e E_{1}+f_{1}\left(v_{1}\right)+f_{12}\left(v_{1}-v_{2}\right)=0 \\
& -n_{2} e E_{2}+f_{2}\left(v_{2}\right)+f_{21}\left(v_{2}-v_{1}\right)=0
\end{aligned}
$$

where $f_{i}(v)$ is the frictional force. A detailed calcu- lation leads to an expression of the frictional forces in terms of intra- and inter-conductor relaxation time functions, $\tau_{1}, \tau_{2}, \tau_{12}$, and $\tau_{21}$, these last ones corresponding to the transresistive frictional forces $f_{12}$ and $f_{21}$. In the linear limit $v_{1}$ and $v_{2}$ are small as compared with natural scales of the systems (like, for instance, the sound velocity), and $T_{1}=T_{2}=T$, where $T$ is the temperature of the heat bath. In consequence the above equations can be enormously simplified:

$$
\begin{aligned}
& -n_{1} e E_{1}-n_{1} \frac{m^{*}\left(v_{1}-v_{2}\right)}{\tau_{12}}-n_{1} \frac{m^{*} v_{1}}{\tau_{1}}=0 \\
& -n_{2} e E_{2}+n_{2} \frac{m^{*}\left(v_{1}-v_{2}\right)}{\tau_{21}}-n_{2} \frac{m^{*} v_{2}}{\tau_{2}}=0,
\end{aligned}
$$

In the case of open circuit $\left(v_{2}^{o}=0, E_{2}^{o} \neq 0\right)$,

$$
\begin{gathered}
-n_{1} e E_{1}^{o}-n_{1} \frac{m^{*} v_{1}^{o}}{\tau_{12}}-n_{1} \frac{m^{*} v_{1}^{o}}{\tau_{1}}=0 \\
-n_{2} e E_{2}^{o}+n_{2} \frac{m^{*} v_{1}^{o}}{\tau_{21}}=0,
\end{gathered}
$$

and

$$
\frac{n_{1}}{\tau_{12}}=\frac{n_{2}}{\tau_{21}}
$$

since $f_{12}=-f_{21}$.

For the case of closed circuit $\left(E_{2}^{c}=0, v_{2}^{c} \neq 0\right)$, however,

$$
\begin{gathered}
-n_{1} e E_{1}^{c}-n_{1} \frac{m^{*}\left(v_{1}^{c}-v_{2}^{c}\right)}{\tau_{12}}-n_{1} \frac{m^{*} v_{1}^{c}}{\tau_{1}}=0 \\
n_{2} \frac{m^{*}\left(v_{1}^{c}-v_{2}^{c}\right)}{\tau_{21}}-n_{2} \frac{m^{*} v_{2}^{c}}{\tau_{2}}=0
\end{gathered}
$$

Next, we calculate the ratio between the induced field $E_{2}^{o}$ in an open circuit set-up and the induced current density $j_{2}^{c}=n_{2} e v_{2}^{c}$ in the case of closed circuit. Generally $\tau_{21} \gg \tau_{2}, \tau_{1}$, so, from Eq.(8),

$$
\frac{v_{1}^{c}}{\tau_{21}}=\frac{v_{2}^{c}}{\tau_{2}} \text { or } v_{1}^{c} \gg v_{2}^{c}
$$


If we apply the same electric field in two cases, i.e. $E_{1}^{o}=E_{1}^{c}$, we have $v_{1}^{o} \simeq v_{1}^{c}$ from Eqs.(4) and (7). Comparing this result with Eq.(5), we obtain,

$$
\frac{E_{2}^{o}}{n_{2} e v_{2}^{c}}=\frac{m^{*}}{n_{2} e^{2} \tau_{2}}
$$

Eq.(10) express the conclusion that the ratio between the induced voltage in open circuit and the induced current in closed circuit equals the resistance of system 2, recovering Ohm's law in the Coulomb drag experiment. It means that in the closed circuit set-up an electromotive force appears in circuit 2, and the resistance of system 2 works as an internal resistance. This result is a mere consequence of the conservation of energy and the balance of forces. Therefore, it is, in fact, not surprising that the Ohm's law applies, since the electromotive force equals the induced voltage in the open circuit set-up.

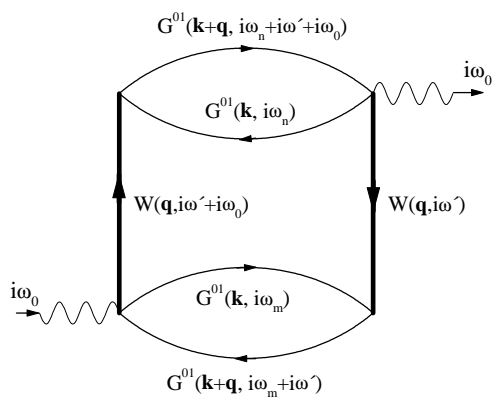

Figure 1. The Feynmann diagram for the drag resistivity.

Most works on Coulomb drag are made in the linear regime, in such a way that the temperature is assumed to be the same for the two subsystems, and equal to that of the heat bath. It is well-known, however, that hot electron effects occur in semiconductor microstructures, [8] where electrons can be easily heated or cooled by very low electric voltage or photon excitations. In non-equilibrium situations, as it is the case in a Coulomb drag experiment, there is no reason to assume, from the very beginning, the two system at the same temperature of the heat bath. Following the suggestion of L. Zheng and A. H. MacDonald[9], we adopt the renormalization of the Coulombic interaction between electron gases 1 and 2, as shown in Fig. 1. For small drift velocities, the drag resistivity becomes[10]:

$$
\begin{aligned}
& R_{d}=\frac{1}{2 \pi^{3} e^{2} n_{1} n_{2}} \int_{0}^{\pi / 2} d \theta \cos ^{2} \theta \int_{0}^{\infty} q^{3} d q \int_{0}^{\infty} d \omega \\
& \left\{\frac{\hat{\Pi}_{2}^{2}\left(\mathbf{q}, \omega, T_{2}\right)}{T_{2} \sinh ^{2}\left(\omega / 2 T_{2}\right)}+2\left[\operatorname{coth}\left(\omega / 2 T_{1}\right)-\operatorname{coth}\left(\omega / 2 T_{2}\right)\right]\right.
\end{aligned}
$$

$$
\left.\frac{\partial}{\partial \omega} \hat{\Pi}_{2}^{2}\left(\mathbf{q}, \omega, T_{2}\right)\right\}\left|\frac{U_{12}(\mathbf{q}, \omega)}{\epsilon_{T}(\mathbf{q}, \omega)}\right|^{2} \hat{\Pi}_{2}^{1}(\mathbf{q}, \omega, T)
$$

where $\hat{\Pi}_{2}^{1(2)}(\mathbf{q}, \omega, T)$ is the (real)immaginary part of the polarization, $U_{12}(\mathbf{q}, \omega)$ is the renormalized interaction potential between gases 1 and 2 , and $\epsilon_{T}(\mathbf{q}, \omega)$ is the total dielectric function. In our calculation the real (virtual) phonon contribution comes from the imaginary (real) part of of the electro-phonon coupling matrix, and the contribution of plasmons and electron-phonon collective modes come from the zeroes of the real part of dielectric function. The contributions of the longitudinal acoustic (LA) and the transversal acoustic (TA) phonons via deformation potential and piezoelectric interaction, are fully included. The mean free times, $\tau_{L O_{p h}}$ and $\tau_{T O_{p h}}$, are used as fitting parameters for both the LA and the TA branches. The parameters used for GaAs are $\kappa_{s}=12.9, d_{1}=5.31 \mathrm{~g} / \mathrm{cm}^{3}, \Xi=14 \mathrm{eV}, \mathrm{m}^{*}=$ $0.067, v_{s l}=5.29 \times 10^{3} \mathrm{~m} / \mathrm{s}, e_{14}=1.41 \times 10^{19} \mathrm{~V} / \mathrm{m}$, and $v_{s t}=2.48 \times 10^{3}$ for the dielectric constant, density, deformation potential, electron effective mass, longitudinal sound velocity, piezoelectric constant and transversal sound velocity, respectively. The numerical calculation is performed for a structure of two $200 \AA$ wide quantum wells, with a $300 \AA$ barrier between them.

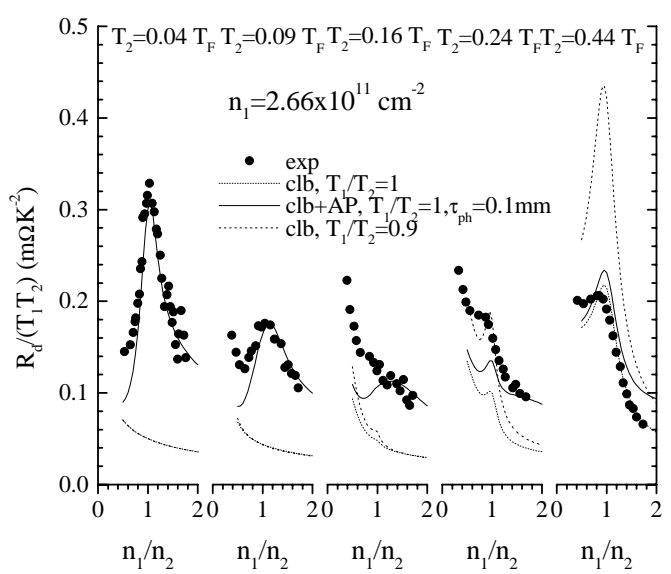

Figure 2. Drag resistivity $R_{d} / T_{1} T_{2}$ is shown as function of driving/drag electron density $n_{1} / n_{2}$ at different normalized drag temperature $T_{2} / T_{F}=0.04,0.09,0.16,0.24,0.44$ and driving electron density $n_{1}=2.66 \times 10^{11} \mathrm{~cm}^{-2}$.

Fig. 2 shows the drag resistivity $R_{d} /\left(T_{1} T_{2}\right)$ in equilibrium cases (solid curves) as a function of the ratio $n_{1} / n_{2}$ of the electron densities at different drag temperatures, $T_{2}=0.04,0.09,0.16,0.24$, and $0.44 T_{F}$ in comparison with the experimental results. The mean free paths of the LA and TA phonons is assumed to be $l_{p h}=0.01 \mathrm{~cm}$. We found that the acoustic phonon enhances the drag resistivity at large $n_{1} / n_{2}$. It can be expected that the inclusion of local field effects will provide good fittings between theoretical and experimental results,[11] except at temperature $T_{2}=0.44 T_{F}$. The 
results with bare Coulomb interaction is shown in equilibrium cases by dotted curves, and in non-equilibrium cases with $T_{1}=0.9 T_{2}$ by dashed curves.

In conclusion, we have studied the momentum transfer rate between two nearby separated electron gases, which are coupled via the Coulomb interaction and phonon mediated interaction. We focused on the non-equilibrium configuration, and a theory was developed to describe the frictional drag force felt by one electron gas as a result of the relative drift of the other, taking into account the possibility of difference on the subsystems temperatures. It was found that a cooler (hotter) driving electron gas greatly enhances (decreases) the frictional force caused by plasmons. This behavior results from the fact the plasmons locate in a region of the $\omega-k$ space where the correlation function shows a nonlinear frequency dependence, and they modifies little the acoustic phonons force because the latter are important mainly in the region of the energy-momentum space where the correlation function is linear with the frequency.

Acknowledgments This work was partially supported by FAPERJ and $\mathrm{CNPq}$ in Brazil.

\section{References}

[1] M. B. Progrebinskii, Fiz. Tekh. Poluprovodn. 11, 637 (1977) [Sov. Phys. Semicond. 11, 372 (1977)].
[2] P. J. Price, Physica B 117, 750 (1983).

[3] P. M. Solomon, P. J. Price, D.J. Frank, and D. C. L. Tulipe, Phys. Rev. Lett. 63, 2508 (1989).

[4] T. J. Gramila, J. P. Eisenstein, A. H. MacDonald, L. N. Pfeiffer, and K. W. West, Phys. Rev. Lett. 66, 1216 (1991); Phys. Rev. B 47, 12957 (1993); Physica B 197, 442 (1994).

[5] U. Sivan, P. M. Solomon, and H. Shtrikman, Phys. Rev. Lett. 68, 1196 (1992).

[6] X.L. Lei and N. J. M. Horing, in Physics of Hot Electron Transport in Semiconductors, edited by C. S. Ting (World Scientific, Singapore, 1992).

[7] H. L. Cui, X. L. Lei, and N. J. M. Horing, Superlattices and Microstructures 13, 221 (1993).

[8] I. C. da Cunha Lima, X. F. Wang and X. L. Lei, Phys. Rev. B 55, 10681(1997); X. F. Wang and X. L. Lei, ibid. 49, 4780(1994).

[9] L. Zheng and A. H. MacDonald, Phys. Rev. B 48, 8203 (1993).

[10] X. F. Wang, and I. C. da Cunha Lima, Phys. Rev. B (2001) (to appear).

[11] N. P. R. Hill, J. T. Nichols, E. H. Linfield, M. Pepper, D. A. Richie, and G. A. Jones, Phys. Rev. Lett 78, 2204 (1997). 